\title{
Therapeutic Potential of Exosomes in Pulmonary Fibrosis
}

\author{
Linshen $\mathrm{Xie}^{1}$ and Ye Zeng ${ }^{2 *}$ \\ ${ }^{1}$ West China School of Public Health and West China Fourth Hospital, Sichuan University, Chengdu, China, ${ }^{2}$ Institute of \\ Biomedical Engineering, West China School of Basic Medical Sciences and Forensic Medicine, Sichuan University, Chengdu, \\ China
}

Pulmonary fibrosis is closely associated with the recruitment of fibroblasts from capillary vessels with damaged endothelial cells, the epithelial mesenchymal transition (EMT) of type II alveolar epithelial cells, and the transformation of fibroblasts to myofibroblasts. Recent studies suggest that EMT is a key factor in the pathogenesis of pulmonary fibrosis, as the disruption of EMT-related effector molecules can inhibit the occurrence and development of PF. With the numerous advancements made in molecular biology in recent years, researchers have discovered that exosomes and their cargos, such as miRNAs, IncRNAs, and proteins, can promote or inhibit the EMT, modulate the transformation of fibroblasts into myofibroblasts, contribute to the proliferation of fibroblasts and promote immunoregulatory and mitochondrial damage during pulmonary fibrosis. Exosomes are

OPEN ACCESS

Edited by: Raffaele Strippoli, Sapienza University of Rome, Italy

Reviewed by: Alice Conigliaro, University of Palermo, Italy Inmaculada Navarro-Lérida, Spanish National Centre for Cardiovascular Research, Spain

*Correspondence: Ye Zeng

ye@scu.edu.cn; yezeng@vip.163.com

Specialty section: This article was submitted to Inflammation Pharmacology, a section of the journal

Frontiers in Pharmacology

Received: 03 August 2020 Accepted: 12 November 2020 Published: 04 December 2020

Citation:

Xie L and Zeng Y (2020) Therapeutic Potential of Exosomes in Pulmonary Fibrosis. Front. Pharmacol. 11:590972. doi: 10.3389/fphar.2020.590972 key factors regulating the differentiation of bone marrow mesenchymal stem cells (BMSCs) into myofibroblasts. Interestingly, exosomes derived from BMSCs under pathological and physiological conditions may promote or inhibit the EMT of type II alveolar epithelial cells and the transformation of fibroblasts into myofibroblasts to regulate pulmonary fibrosis. Thus, exosomes may become a new direction in the study of drugs for the treatment of pulmonary fibrosis.

Keywords: exosome, epithelial mesenchymal transition, pulmonary fibrosis, bone marrow mesenchymal stem cell, type II alveolar epithelial cell, myofibroblast

\section{INTRODUCTION}

Pulmonary fibrosis (PF) is a pathological process that occurs after lung tissue damage and is the final manifestation of many lung diseases. PF has been shown to be a complex pulmonary interstitial disease caused by multiple factors, such as genetic abnormalities, autoimmunity, occupational exposure, trauma, radioactive element exposure, pathogenic microorganism infection, and drug administration (King et al., 2011; Meyer, 2017). Patients with PF typically have progressive dyspnea accompanied by irritating dry cough, with respiratory functions that will deteriorate with the aggravation of PF, eventually leading to respiratory failure and death (Thannickal et al., 2004). The median survival time of PF patients is only 3-5 years after diagnosis (Hopkins et al., 2016; Yanagihara et al., 2020). Diffuse pneumonia appears in the early stage of PF, while an excessive proliferation of fibroblasts and the deposition of extracellular matrix (ECM) appear during the later period (Gross and Hunninghake, 2001; Kinoshita and Goto, 2019).

Silicosis is an occupational disease with the highest morbidity and mortality in China and worldwide. Silicosis, which has a long incubation period and is difficult to detect, can still occur after an individual is no longer subject to silica dust exposure (Liu et al., 2013). From 1999-2018 in the USA, pneumoconiosis deaths among coal workers associated with pneumoconiosis and silicosis 
decreased by 69.6 and 53.0\%, respectively (Bell and Mazurek, 2020). As reported in the Statistical Bulletin of Health Development in China at 2019, more than 19,428 cases of occupational diseases have been reported in China, of which more than 15,898 are cases of pneumoconiosis, with silicosis accounting for $81.8 \%$ of these cases. Silicosis is associated with a $61 \%$ overall increase in lung cancer risk (Liu et al., 2013) and is characterized by diffuse fibrosis of lung tissue, which seriously affects the quality of life survival time of patients (Barnes et al., 2019; Hoy and Chambers, 2020). Due to its unclear pathogenesis and a lack of clinically effective therapeutic drugs, the development of new methods and drugs for the prevention and treatment of PF has become a hot topic (Worthington and Hagood, 2020). Thus, there is an urgent need to identify effective intervention targets and develop methods and strategies to control and delay PF.

It is currently believed that the pathogenesis of PF can be divided into following three processes (Wynn, 2011; Kropski and Blackwell, 2019; Suryadevara et al., 2020): 1) susceptibility processes, including genetic mutations, environmental exposure, and aging processes, which make individuals susceptible to PF; 2) activation processes, including transforming growth factor- $\beta$ (TGF- $\beta$ ) activation, fibroblast recruitment from capillary vessels with damaged endothelial cells, the epithelial mesenchymal transition (EMT), and the unfolded protein response (UPR) activation, which promote the progress of fibrosis.; and 3) progressive processes, including pathological fibroblast differentiation, endothelial cell death with vessel loss and new vessel formation, matrix deposition and remodeling, increased stiffness, and profibrotic epigenetic changes in fibroblasts and epithelial cells. The EMT of damaged lung epithelial cells causes continuous mesenchymal cell activation and matrix remodeling, accelerating the production of excessive ECM deposition and the formation of fibrous scar tissue, which eventually leads to lung failure.

Recent studies have suggested that injury to type II alveolar epithelial cells, the interaction between alveolar epithelial cells and fibroblast, and the EMT play crucial roles in the pathogenesis of PF (Kropski and Blackwell, 2019; Yanagihara et al., 2020). EMT promotes fibrosis by activating signaling pathways associated with this process. The disruption of EMTrelated effectors can inhibit the occurrence and development of PF (Kropski and Blackwell, 2019). With the continuous advancements in molecular biology in recent years, researchers have discovered that some regulatory microRNAs (miRNAs) and exosomes are closely associated EMT (Xu et al., 2019; Feng et al., 2020). Exosomes are present in various body fluids, such as blood, urine and sputum, and they can be used as potential biomarkers and treatments for different diseases, including PF (Keller et al., 2011; Guiot et al., 2019; Fukushima et al., 2020; Khalaj et al., 2020). The goal of this article is to review recent research progress on the role of EMT in the pathogenesis of PF and the potential role of exosomes and their specific cargoes.

\section{Exosomes and Epithelial Mesenchymal Transition}

Exosomes are extracellular vesicles (EVs) that have become a hot topic in the field of EV research (Colombo et al., 2014; Cocozza et al., 2020). Exosomes are extracellular nanovesicles with a lipid bilayer structure that are released by cells through efflux and have diameters ranging from 30-150 nm (van Niel et al., 2018; Mathieu et al., 2019). Exosomes are nanoscale vesicles secreted by cells under physiological or pathological conditions (Wen et al., 2017; Stahl and Raposo, 2019). Exosomes are secreted from cells via exocytosis and absorbed by target cells, resulting in the transfer of biological signals (Yang et al., 2018). Many types of cells have been shown to secrete exosomes, such as B cells, epithelial cells, fibroblasts, and a variety of tumor cells (Mehryab et al., 2020; Moghiman et al., 2020; Peng et al., 2020). As important mediators of intercellular communication, the functions of exosomes are dependent on their cargo profile, which includes miRNAs, messenger RNAs, DNA, and proteins that contribute to both physiological functions and the pathophysiologies of a variety of diseases, including PF (Colombo et al., 2014; Akbari and Rezaie, 2020; Cocozza et al., 2020; Khalaj et al., 2020).

EMT is a process in which fully differentiated epithelial cells gradually transform into mesenchymal cells and lose their original function after exposure extracellular factors (Nieto et al., 2016). EMT is an important mechanism leading to the generation of myofibroblasts. During the EMT process, epithelial cells undergo significant changes in transcriptional regulation, cytoskeleton shape, adhesion, and ECM components (Singh et al., 2018). Under normal conditions, epithelial cells are tightly connected through mechanisms promoting adhesion between cells. During EMT, cells separate from neighboring cells and migrate to adjacent tissues, altering their function. During EMT, the expression of epithelial cell markers, such as E-cadherin, cytoplasmic zonula occludens protein 1 (ZO-1), and $\alpha$-catenin are decreased, while that of fibroblast specific protein 1 (FSP-1), $\mathrm{N}$-cadherin, vimentin, fibronectin and $\alpha$-smooth muscle actin (SMA) are increased (Singh et al., 2018). Based on differences in biological environments, EMT can be divided into three subtypes (Nieto et al., 2016). Type I EMT is associated with embryonic development, including processes such as embryonic differentiation, organ formation, and nervous system differentiation. Type II EMT occurs during tissue repair, regeneration, and organ fibrosis. Under normal circumstances, type II EMT will cease when the inflammatory reaction is inhibited, but it will continue to aggregate fibroblasts if the injury and inflammation are not improved. Type III EMT is involved in tumor metastasis. During this process, the deformation, migration, and anti-apoptosis abilities of epithelial cells are enhanced and are associated with a lack of cell polarity, a weakening of cell adhesion, cytoskeletal remodeling, and the disappearance of other cell movement elements. Compared to type I and type III EMT, type II EMT is caused by persistent injury and inflammation, which is an important organ fibrosis process (Xu et al., 2019). 
TABLE 1 | Exosomal cargos promote or inhibit the EMT of lung epithelial cells.

\begin{tabular}{|c|c|c|c|}
\hline Exosomal cargo & Parental cells & Recipient cells & References \\
\hline \multicolumn{4}{|l|}{ Inducer } \\
\hline miR-210 & Cancer-related fibroblasts & Non-small cell lung cancer cells (H1975 and A549) & (Yang et al., 2020) \\
\hline Snail1 & Cancer-related fibroblasts & Lung cancer cells & (You et al., 2019) \\
\hline miR-499a-5p & A549, SPC-A-1-BM & A549, SPC-A-1-BM & (He et al., 2019b) \\
\hline miR-200c, miR-205 & Mesenchymal phenotype A549 & $\mathrm{N} / \mathrm{A}$ & (Tang et al., 2018) \\
\hline ZEB1 mRNA & Mesenchymal bronchial epithelial cells & Recipient epithelial cells & (Lobb et al., 2017) \\
\hline $\mathrm{miR}-23 \mathrm{a}$ & TGF- $\beta 1$-induced A549 cells & A549 & (Kim et al., 2016) \\
\hline miR-193a-3p, miR-210-3p, miR-5100 & Hypoxic BMSCs & Lung epithelial cancer cells H358, A549, and H460 & (Zhang et al., 2019) \\
\hline $\mathrm{N} / \mathrm{A}$ & Human umbilical cord MSCs & A549 & (Zhao et al., 2018) \\
\hline PD-L1 & A549, H1299 & A549, H1299 cells & (Hong et al., 2020) \\
\hline miR-21 & Bronchial epithelial cells (BEAS-2B) & Bronchial epithelial cells (BEAS-2B) & (He et al., 2019a) \\
\hline \multicolumn{4}{|l|}{ Suppressor } \\
\hline miR-26a & Human airway epithelial cell line $16 \mathrm{HBE}$ & - & (Tang et al., 2018) \\
\hline
\end{tabular}

Exosomes are closely associated with EMT, a process that involves the disappearance of epithelial cell characteristics and the appearance of a mesenchymal cell phenotype and the ability to migrate (Singh et al., 2018). This is a complex process that involves a number of changes, such as cytoskeletal remodeling and reduced E-cadherin levels. Exosomes can promote or inhibit EMT, thereby contributing to the formation of the tumor microenvironment and various pathological processes, such as tumor metastasis, kidney injury, and fibrosis (Syn et al., 2016; Xiao et al., 2016; Conigliaro and Cicchini, 2018). Previous studies have shown that exosomes also play a role in immune regulation, the transition of epithelial cells into fibroblasts and mesenchymal cells (EMT), and the transformation of fibroblasts to myofibroblasts ( $\mathrm{Hu}$ et al., 2020; Li et al., 2020b; Qin et al., 2020). Table 1 summarizes the exosomal cargos that promote or inhibit the EMT of lung epithelial cells.

\section{Exosomal Cargos and Epithelial Mesenchymal Transition Promotion}

Exosomes exert their functions through the cargos they harbor and can carry molecules that promote EMT and act directly on target cells to mediate EMT. Exosomes contain a variety of molecules that can regulate cell function and promote EMT, such as matrix metalloproteinases (MMPs), hypoxia inducible factor-1a (HIF-1a), and so on (Hu et al., 2020; Kim et al., 2016). MiRNAs are major components of exosomes (Zeng et al., 2019). These molecules can bind to and regulate target mRNAs by inhibiting their translation or promoting their degradation and are widely involved in cell proliferation, apoptosis, development, differentiation, angiogenesis, and fibrosis (Kadota et al., 2020; Yao et al., 2020). Exosomes secreted by cancer-related fibroblasts and harboring miR-21, mir-378e and miR-143 can induce EMT in different breast cancer cell lines, and miR-21 can also promote EMT in renal tubular epithelial cells (Donnarumma et al., 2017). MiR-210 in exosomes derived from cancer-associated fibroblasts can induce the EMT of non-small cell lung cancer cells (H1975 and A549) via the PTEN/PI 3 K/AKT pathway (Yang et al., 2020). The level of SNAI1 in exosomes derived from cancer-related fibroblasts is associated with the level of SNAI1 in cancer-related fibroblasts, which is crucial for inducting EMT in lung cancer cells (You et al., 2019). The level of exosomal miR-499a-5p is associated with the level of miR-499a-5p and the EMT of the human lung cancer and human lung adenocarcinoma cell lines A549 SPC-A-1-BM (He et al., 2019b). MiR-23b-enriched exosomes from TGF- $\beta 1$-induced A549 cells can promote the EMT of A549 (Kim et al., 2016), while miR-23a can promote TGF- $\beta 1$-induced EMT in lung cancer cells (Cao et al., 2012). Exosomes derived from mesenchymal bronchial epithelial cells but not epithelial bronchial epithelial cells contain ZEB1 mRNA and can induce the EMT of recipient epithelial cells (Lobb et al., 2017). MiR-200c and miR-205 levels in exosomes from invasive cells (mesenchymal phenotype A549) are significantly decreased compared to those observed in noninvasive cells (16HBE cells) (Tang et al., 2018), with both miR-200c and miR-205 being EMT inducers (Tellez et al., 2011).

EMT induced by exosomal cargos is associated with immune regulation. The knockdown of circular RNA (cirRNA) cirCPA4 can inhibit the growth, mobility and EMT of A549 cells by inhibiting exosomal PD-L1 levels (Hong et al., 2020). The exposure of macrophages to apoptotic cells inhibits TGF- $\beta 1$-induced EMT in primary mouse lung alveolar epithelial cells in vivo and in vitro (Yoon et al., 2016). The results of a recent study further suggest that conditioned medium from macrophages exposed to apoptotic cancer cells inhibits the TGF- $\beta 1$-induced EMT of A549 cells (Kim et al., 2019). Cigarette smoke extract was shown to induce the production of EVs with low miR-21 levels from bronchial epithelial cells to ameliorate EMT in chronic obstructive pulmonary disease by alleviating M2 macrophage polarization ( $\mathrm{He}$ et al., 2019a). Reversing the expression of EMT mediators in exosomes or suppressing the release of EMT-related exosomes would be a viable therapy for lung fibrosis. A recent study showed that aspirin reversed the EMT of nasopharyngeal carcinoma cells by promoting miR-203 expression and repressed exosomal latent membrane protein 1 (LMP1) secretion in Epstein-Barr virus-positive cells (Zuo et al., 2019). Exosomal LMP1 promotes the EMT potential of Epstein-Barr virusnegative cells. 


\section{Exosomal Cargos and Epithelial Mesenchymal Transition Inhibition}

Exosomes can also inhibit EMT through their cargos. Exosomal miR-1255b-5p can suppress the EMT of colorectal cancer cells by inhibiting Wnt/ $\beta$-catenin activation (Zhang et al., 2020), while exosomal miR-34c from mesenchymal stem cells can inhibit the EMT of nasopharyngeal carcinoma cells by targeting $\beta$-catenin (Wan et al., 2020). In another study, MSC-derived exosomal miR3940-5p was shown to inhibit the EMT of colorectal cancer by targeting integrin alpha6 (ITGA6) (Li et al., 2020d), while exosomal miRNA-215-5p in adipose-derived stem cells inhibited the EMT of podocytes by inhibiting ZEB2 (Jin et al., 2020). MiR-26a levels in exosomes from invasive cells (mesenchymal phenotype A549) are significantly increased compared to those in exosomes from noninvasive cells (16HBE cells) (Tang et al., 2018). MiR-26a is an EMT suppressor (Liang et al., 2014). However, the results of recent studies have indicated that EMT-inhibiting exosomal cargos are almost miRNAs, and whether exosomal proteins, lipids, and even long non-coding RNA (lncRNAs) can inhibit EMT, and their associated mechanisms, remain to be explored in depth.

\section{Regulation of Epithelial Mesenchymal Transition by Exosomes Derived From Mesenchymal Stem Cells}

Exosomes derived from mesenchymal stem cells (MSCs) has been shown to regulate EMT. Exosomal miRNAs, including miR193a-3p, miR-210-3p and miR-5100 derived from hypoxic bone marrow mesenchymal stem cells (BMSCs) activate STAT3 signaling and enhance the EMT of lung epithelial cancer cells (Zhang et al., 2019). Human umbilical cord MSCs promote the EMT, invasion, and migration of A549 lung cancer cells via MSC-derived exosomes, while silencing TGF- $\beta 1$ expression in MSCs can inhibit the EMT-promoting effect of MSCs on A549 cells via MSC-derived exosomes (Zhao et al., 2018). However, human umbilical cord MSC-exosomal miR3940-5p inhibits the invasion and EMT of colorectal cancer cells (Li et al., 2020d). Exosomes derived from human umbilical cord MSCs can suppress the EMT and liver fibrosis (Li et al., 2013). Therefore, the effects of exosomes derived from different MSCs on EMT is dependent on the source of MSCs under specific conditions, and the molecules in MSC-derived exosomes associated with EMT remain unclear.

\section{Primary Epithelial Mesenchymal Transition Signaling Pathways Wnt Signaling Pathway}

The Wnt signaling pathway comprises three primary pathways: the $\mathrm{Wnt} / \beta$-catenin, Wnt/JNK, and $\mathrm{Ca}^{2+}$-mediated $\mathrm{Wnt} / \mathrm{Ca}^{2+}$ signaling pathways (Kohn and Moon, 2005; Teeuwssen and Fodde, 2019). The results of a literature search revealed 19 types of Wnt genes in the human genome that encode functionally distinct proteins (Wodarz and Nusse, 1998). Among them, Wnt2, Wnt3, and Wnt8b are involved in classic $\mathrm{Wnt} / \beta$-catenin signaling pathways, while Wnt6, Wnt7b, and
Wnt10a are involved in nonclassical Wnt signaling pathways, including the $\mathrm{Wnt} / \mathrm{JNK}$ and $\mathrm{Ca}^{2+}$-mediated $\mathrm{Wnt} / \mathrm{Ca}^{2+}$ pathways. The classic Wnt/ $\beta$-catenin signaling pathway is an important pathway that regulates the occurrence and development of EMT (Teeuwssen and Fodde, 2019) and comprises the secreted protein Wnt family, secreted frizzled-related protein (sFRP), protein phosphatase 2A (PP2A), transmembrane receptor frizzled protein $\mathrm{Fz}$, casein kinase (CK1), the Dickkopf protein (DKK)

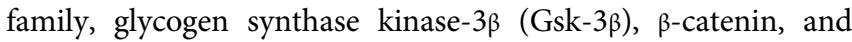
adenomatous polyposis coli (APC).

When Wnt is activated, the extracellular domain of the Wnt receptor $\mathrm{Fz}$ binds to Wnt, after which $\beta$-catenin cannot be degraded in the cytoplasm. $\beta$-Catenin functions as a carrier to mediate the formation of the E-cadherin and $\alpha$-catenin complex, inhibiting the activation of E-cadherin. After accumulating to a specific level, $\beta$-catenin is translocated to form a complex with nuclear transcription factor (TCF/LEF) to activate downstream target genes such as snail and slug, thereby promoting EMT (Teeuwssen and Fodde, 2019). After Wnt signal activation, GSK$3 \beta$ activity is inhibited, which decreases the degradation of $\beta$-catenin, inducing cell morphology changes and regulating the EMT. The Wnt/ $\beta$-catenin signaling-mediated induction of EMT is involved in various cancers, such as prostate cancer and ovarian cancer (Teeuwssen and Fodde, 2019; Yeh et al., 2019), as well as organ fibrosis such as renal fibrosis and liver fibrosis (Edeling et al., 2016; Wang et al., 2018a).

Exosomes released from colon cancer cells with high levels of the Wnt receptor frizzled 10 protein (FZD10) can stimulate EMT activation to reprogram normal colonic epithelial cells (Scavo et al., 2020). Cancer stem cell-like cell-derived exosomal lncRNA DOCK9 antisense RNA2 (AS2) can activate the Wnt/ $\beta$-catenin pathway to induce EMT and promote the progression of papillary thyroid carcinoma (Dai et al., 2020). LncRNA ADAMTS9-AS1 can suppress the EMT of colorectal cancer cells and is markedly reduced in serum exosomes from colorectal cancer patients ( $\mathrm{Li}$ et al., 2020c). Therefore, exosomes can regulate the EMT via the Wnt signaling pathway. However, how $\mathrm{Wnt} / \beta$-catenin signaling regulates exosomes and contributes to $\mathrm{PF}$ remains to be investigated.

\section{Rho-ROCK Signaling Pathway}

The Rho-ROCK signaling pathway is commonly used by various types of cells (Johan and Samuel, 2019). Rho family proteins have guanosine triphosphate (GTP) enzyme activity and are also known as RhoGTPases, members of which belongs to the Ras superfamily of GTP binding proteins. There are more than 20 different Rho proteins in mammalian cells. Rho family proteins are believed to regulate multiple biological processes including cytoskeleton remodeling, cell morphology changes, cell proliferation, and adhesion through intracellular signaling pathways (Porazinski et al., 2020). Rho-associated coil forming protein kinase (ROCK) is the downstream target effector of Rho. ROCK receives the activation signal transmitted by Rho, which promotes the phosphorylation of its amino acid domain and leads to a series of phosphorylation and dephosphorylation reactions. Rho-ROCK signaling is a key regulator of actomyosin-mediated contractility, cell shape, and cytoskeletal arrangement, thereby 
affecting cell functions such as cell proliferation, differentiation, motility, and adhesion. The inhibition of Rho/ROCK signaling can downregulate the expression of EMT-related molecules (Liu et al., 2020). The primary proximal tubule cells in a hypertensive chronic kidney disease model showed upregulation of RhoROCK signaling accompanied by EMT, which was characterized by the development of spindle shape morphology, gene expression changes in EMT markers (collagen type I alpha 3, MMP9, BMP7, and occludin) and increased $\mathrm{N}$-cadherin and vimentin expression (Jia et al., 2015). Incubating human bronchial epithelial cells (BECs) with silica in vitro induced the de novo expression of a-SMA and vimentin and decreased that of E-cadherin. In addition, silica treatment of human BECs could result in Rho activation. In another study, an inhibitor of a Rho effector protein could upregulate the E-cadherin expression while attenuating a-SMA and vimentin expression in silica-stimulated cells. All of these results demonstrated that activation of the Rho-ROCK signaling pathway is most likely involved in silica-induced EMT in human BECs (Hu et al., 2013).

Liver fibrosis has been shown to be reduced by the administration of the ROCK1 inhibitor fasudil, which was accompanied by reduced serum levels of EVs (Hirsova et al., 2016). However, the link between the Rho-ROCK signaling pathway and exosomes in PF remains poorly studied.

\section{Transforming Growth Factor- $\beta /$ Smad Pathway}

TGF- $\beta 1$ is considered to be the most important factor in PF. TGF$\beta 1$ not only induces alveolar EMT but also promotes the differentiation of bone marrow mesenchymal stem cells (BMSCs) into myofibroblasts (Li et al., 2020a; Qian et al., 2020; Yang et al., 2012). TGF- $\beta 1$ levels were shown to be significantly reduced during the BMSC-conditioned mediummediated inhibition of silicosis (Wang et al., 2018b). After the body is stimulated by inflammatory or injury factors, such as endotoxin and reactive oxygen species, a variety of cells (including type II alveolar epithelial cells) produce TGF- $\beta 1$ (Kumari et al., 2017).

The TGF- $\beta$ superfamily comprises 25 proteins, including TGF- $\beta$ s, activins, inhibins, and growth differentiation factors (GDFs). GDFs play an important role during embryonic development, cell division, organ formation, and tissue repair. TGF- $\beta 1$ is one of the most important TGF- $\beta$ subtypes and can be secreted by endothelial cells, $\mathrm{T}$ lymphocytes, B lymphocytes, and fibroblasts and is expressed in various organs and tissues (Hayashi and Sakai, 2012; Lichtman et al., 2016). It plays a role in cell division, proliferation, differentiation, apoptosis, and migration. TGF$\beta 1$ stimulates the production of ECM and promotes the degradation of ECM. TGF- $\beta$ receptors (T $\beta$ Rs) are widely expressed in various tissues. There are three subtypes of TGF- $\beta$, including TGF- $\beta 1$, TGF- $\beta 2$, and TGF- $\beta 3$, among which $\beta 1$ and $\beta 2$ are signal transduction receptors with structures comprising extracellular, transmembrane, and intramembrane regions (Budi et al., 2017; Kang et al., 2009). The extracellular domain of $T \beta R$ is rich in cysteine, which can bind to the ligand and activate the downstream signal cascade. T $\beta$ RI mediates the nuclear localization of Smad3, the induction of a-SMA during the collagen-induced EMT of alveolar epithelial cells, and fibroblast accumulation during idiopathic pulmonary fibrosis (IPF) (DeMaio et al., 2012). T $\beta$ RII knockout can inhibit the normal nuclear localization of $\beta$-catenin, causing cell death, cell cycle arrest, increased of fibrotic factor expression, and renal fibrosis (Nlandu-Khodo et al., 2017). TßRII knockdown can also promote PF, while hydroxysafflor yellow A can inhibit the TGF- $\beta 1$-mediated activation of fibrotic changes by targeting T $\beta$ RII in human fetal lung fibroblasts (MRC-5) (Pan et al., 2017).

TGF- $\beta 1$ signaling pathways primarily comprise the TGF- $\beta 1 /$ Smad signaling pathway and the Smad-independent signaling pathway (Shi and Massagué, 2003; Macias et al., 2015). Smad is a transcriptional coordination factor of the TGF- $\beta$ superfamily present in the cytoplasm and mediates TGF- $\beta$ signaling in the nucleus. At present, 9 Smad proteins have been identified that are divided into three subtypes (Sun et al., 2014; Ng-Blichfeldt et al., 2019). Membrane receptor-regulated Smads (R-Smads) include Smad1, Smad2, Smad3, Smad5, and Smad8, among which Smad2 and Smad 3 bind to TGF- $\beta 1$ to form a transcription complex that binds to a specific region of the downstream target gene and then participates in TGF- $\beta$ signal transduction. Common pathway Smads (co-Smads) include Smad4 and Smad10, where Smad4 binds R-Smad to form a heterologous complex and enter the nucleus to participate in transcription al regulation. Inhibitory Smads (I-Smads) include Smad 6 and 7, which play a negative role in regulating the TGF- $\beta 1$ signal transduction pathway. The TGF- $\beta$ signaling pathways include the classical and nonclassical Smad signaling pathways. The classical Smad signaling pathway is the primary pathway involved in the TGF- $\beta$-mediated induction of fibrosis. The Smad-independent signaling pathway primarily involves the mitogen-activated protein kinase (MAPK) pathway (Du et al., 2020; Luo et al., 2020), which includes three members: p38MAPK, extracellular signalrelated kinase (ERK), and c-Jun N-terminal kinase (JNK). Crosstalk has been shown to occur between the TGF- $\beta 1$ and Wnt/ $\beta$-catenin signaling pathways (Basile and Mehrotra, 2017).

TGF- $\beta 1$ directly activates Smad signaling, which triggers profibrotic gene overexpression. TGF- $\beta 1$-induced EMT had been widely demonstrated in different pulmonary diseases, such as asthma (Ijaz et al., 2014), pulmonary cystic fibrosis (Rout-Pitt et al., 2018), IPF and hypersensitivity pneumonitis (Kiszalkiewicz et al., 2017). In Smad3-deficient mouse models, type I procollagen expression is suppressed, and the hydroxyproline content in the lungs is reduced compared to wild-type mice treated with bleomycin (Zhao et al., 2002). Furthermore, the loss of Smad3 greatly attenuates the morphological fibrotic response to bleomycin in the mouse lung. These results demonstrate that Smad3 contributes to bleomycin-induced lung injury and that Smad3 may serve as a novel target for the potential treatment of lung fibrosis (Zhao et al., 2002).

The exosomal delivery of TGF- $\beta$ contributes to the activation of TGF- $\beta$ signaling under pathological conditions such as tumor and hypoxia, contributing to EMT and myofibroblast differentiation (Ahuja et al., 2020; Chowdhury et al., 2015). 


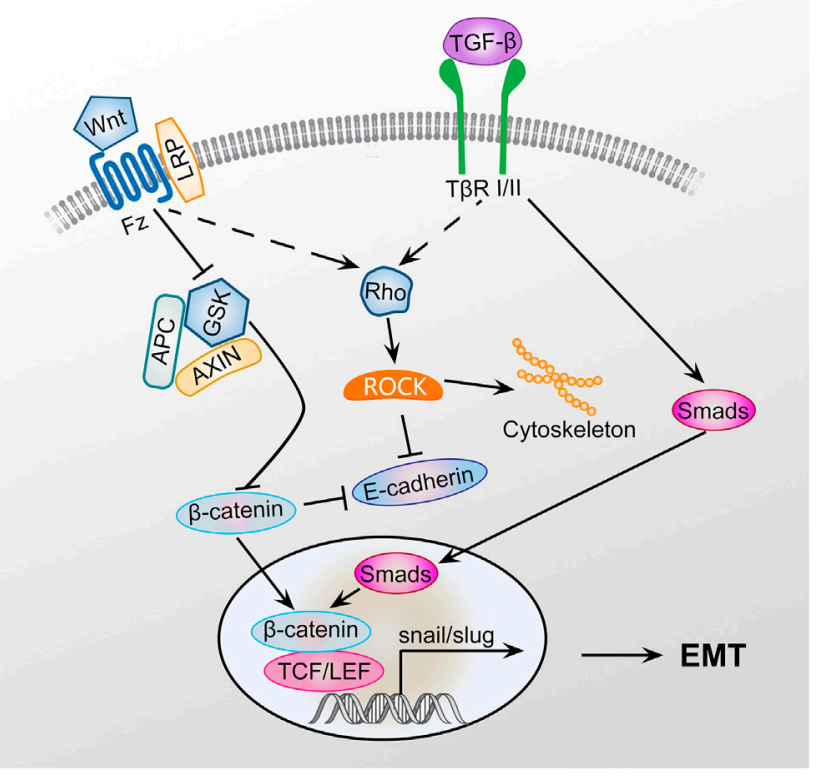

FIGURE 1 | Signaling pathway of EMT. GSK3 $\beta$ is an important negative regulator of the Wnt signaling pathway. Axin can interact with many important members of the Wnt signaling pathway to negatively regulating $\mathrm{Wnt} / \beta$-catenin signaling. Wnt signaling pathway activation inhibits the phosphorylation of GSK3 $\beta$, preventing GSK3 $\beta$ from phosphorylating $\beta$-catenin, leading to the inhibition of $\beta$-catenin degradation. GSK3 $\beta$ phosphorylation can promote axin autophosphorylation, increasing the affinity of axin and $\beta$-catenin and promoting $\beta$-catenin degradation. $\beta$-Catenin can promote the EMT of type II alveolar epithelial cells, promote the transformation of type II alveolar epithelial cells to myofibroblasts, and ultimately promote PF by activating the TGF- $\beta 1$ / Smad and Rho-ROCK signaling pathways.

Exosomes derived from human umbilical cord MSCs can reduce TGF- $\beta$ levels and liver fibrosis in vivo and inhibit EMT in vitro (Li et al., 2013). Similarly, silencing TGF- $\beta 1$ expression in human umbilical cord MSCs can revert the EMT-promoting effect of MSCs on A549 cells via MSC derived exosomes (Zhao et al., 2018). BMSC-derived exosomes can reverse EMT in rabbit endometrial epithelial cells induced by TGF- $\beta 1$, inhibiting endometrial fibrosis (Yao et al., 2019b). Thus, the differences between BMSC-derived exosomes from cells under normal and abnormal conditions such as cancer, hypoxia, and pro-fibrosis should not be neglected.

EMT is a key step in development, wound healing, fibrosis, and cancer that involves a complex network of signaling pathways, including the TGF- $\beta 1 / \mathrm{Smad}$, Rho-ROCK, and Wnt $/ \beta$-catenin pathways (Figure 1). These signal factors can interact with each other through negative or positive feedback loops that forms a closed network (Zhang et al., 2016). EMT induction by exosomes with particulate matter having a diameter of less than $2.5\left(\mathrm{PM}_{2.5}\right)$ may be associated with activation of the TGF- $\beta / \mathrm{Smad}$ and $\mathrm{Wnt} / \beta$-catenin pathways to promote fibrogenesis (Xu et al., 2019). However, the detailed signaling mechanisms involved in exosomal cargo-mediated EMT and their contributions in PF remain unclear.

\section{Exosomes and Transformation of Fibroblasts to Myofibroblasts}

EVs, especially exosomes, can regulate the differentiation of fibroblasts into myofibroblasts (Table 2). EV-associated miR210 from primary human bronchial epithelial cells and lung fibroblasts induced by cigarette smoke extract acts as a paracrine autophagy mediator of myofibroblast differentiation in lung fibroblasts (Fujita et al., 2015). Activated primary human lung fibroblasts can release prostaglandins such as $\mathrm{PGE}_{2}$-enriched exosomes or EVs to inhibit TGF- $\beta 1$-induced myofibroblast differentiation (Lacy et al., 2019), while miR-22 negatively regulates myofibroblast differentiation from lung fibroblasts and ameliorates PF in mice (Kuse et al., 2020).

The number of EVs, especially exosomes, is increased in bronchoalveolar lavage (BAL) fluid from experimental lung fibrosis and patients with IPF and are rich in WNT5A (Martin-Medina et al., 2018). Interestingly, EV-associated WNT5A may be associated with fibroblast activation (MartinMedina et al., 2018). A recent study showed that macrophages are the major secretors of exosomes containing early secreted proinflammatory cytokines in BAL fluid (Ye et al., 2020). Exosomal miR-125a-5p derived from silica-exposed macrophages induces the fibroblast to myofibroblast transition and silicosis (Wang et al., 2020). Exosomes derived from lung spheroid can could attenuate and resolve bleomycin- and silica-induced fibrosis (Dinh et al., 2020) and contain miRNAs in the let-7 and miR99 families as well as numerous growth factors and ECM-related proteins. The identification of crucial molecules in exosomes may benefit the development of effective IPF therapies using secreted EVs (Dinh et al., 2020).

\section{Novel Mechanisms of PF Mediated by Exosomes}

In addition to the EMT and transformation of fibroblasts into myofibroblasts, other mechanisms exist with respect to the modulation of PF by exosomes. MiR-328 derived from M2 macrophages promotes lung fibroblast proliferation and $\mathrm{PF}$ (Yao et al., 2019a). In another study, exosomal miR-142-3p from macrophages was shown to attenuate $\mathrm{PF}$, possibly by reducing the expression of $\mathrm{TGF} \beta \mathrm{I}$ and pro-fibrotic genes in alveolar epithelial cells and lung fibroblasts (Guiot et al., 2020). BMSCs promote an immunoregulatory, anti-inflammatory monocyte phenotype to prevent PF (Mansouri et al., 2019). Exosomal let-7 from menstrual blood-derived endometrial stem cells alleviates lung fibrosis and alveolar epithelial cell damage in mice by regulating reactive oxygen species levels, mitochondrial DNA damage and NLRP3 inflammasome activation (Sun et al., 2019). Lung fibroblast-derived EVs from IPF patients were shown to increase mitochondrial reactive oxygen species levels and associated mitochondrial damage in lung epithelial cells, and these EVs were sown to contain elevated levels of miR-23b-3p and miR-494-3p (Kadota et al., 2020). Thus, the proliferation of fibroblasts, immunoregulatory pathways, and mitochondrial damage are novel mechanisms and promising targets for exosomal PF therapy. 
TABLE 2 | Regulation of lung fibroblast transdifferentiation by exosomes.

\begin{tabular}{|c|c|c|c|}
\hline Exosomal cargo & Parental cells & Effect & References \\
\hline miR-210 (EV) & Cigarette smoke extract-induced human bronchial epithelial cells and lung fibroblasts & Promotion & (Fujita et al., 2015) \\
\hline $\mathrm{PGE}_{2}$ & Human lung fibroblast & Inhibition & (Lacy et al., 2019 \\
\hline miR-22 & PF mice & Inhibition & (Kuse et al., 2020) \\
\hline WNT5A & Bronchoalveolar lavage (BAL) fluid & Promotion & (Martin-Medina et al., 2018) \\
\hline MiR-125a-5p & Silica-exposed macrophages & Promotion & (Wang et al., 2020) \\
\hline Let-7 and miR-99 families & Lung spheroid cell & Inhibition & (Dinh et al., 2020) \\
\hline
\end{tabular}

\section{Therapeutic Potential of BMSCs and Their Exosomes}

The primary manifestation of PF is an abnormal increase in the number of interstitial cells and ECM levels (Worthington and Hagood, 2020). Myofibroblasts are the primary effector cells that synthesize and secrete ECM during PF (Kropski and Blackwell, 2019; Suryadevara et al., 2020; Wynn, 2011). In IPF, the myofibroblasts in fibrosis lesions are primarily derived from the transformation of the original fibroblasts in lung tissue, the migration and differentiation of bone marrowderived fibroblasts in peripheral circulation, and the EMT of local alveolar epithelial cells (Worthington and Hagood, 2020). In renal fibrosis, $35 \%$ of myofibroblasts are derived from bone marrow, which depends on the TGF- $\beta 1$ signaling pathway (LeBleu et al., 2013). BMSCs migrate to the injured liver and differentiate to myofibroblasts via cannabinoid receptor 1 (Wang et al., 2017). Nicotine exposure drives the differentiation of BMSCs into myofibroblasts in the lungs (Sakurai et al., 2018). However, autologous BMSCs treated with hepatocyte growth factor can effectively improve the adverse symptoms of silicosis patients and promote the absorption of silicosis nodules (Liu et al., 2015; Yan et al., 2016). The administration of normal BMSCs to mice with silicosis via tail vein injection can also reduce silicosis (Wang et al., 2018b). In one study, exosomes derived from Wharton's jelly mesenchymal stem/stromal cells (WJMSCs) and BMSCs can modulate lung macrophage phenotypes, restore the lung architecture and decrease fibrosis in hyperoxia $\left(75 \% \mathrm{O}_{2}\right)$ exposed mice, while exosomes derived from human dermal fibroblasts could not (Willis et al., 2018). In another study, BMSCs overexpressing transforming growth factor-beta 3 (TGF- $\beta 3$ ), an inhibitor of myofibroblast proliferation, could promote wound healing and reduce scar tissue formation in a rabbit model ( $\mathrm{Li}$ et al., 2018a). In addition, the transplantation of BMSCs overexpressing miR-124a to diabetic nephropathy rats could mitigate the EMT of podocytes and renal fibrosis (Cai et al., 2020). Granulocyte-colony stimulating factor (G-CSF) can enhance the homing of autologous BMSCs to damaged lung tissue to inhibit bleomycin-induced PF (Zhao et al., 2020). Thus, BMSCs act as a double-edged sword, where BMSCs in PF patients serve as an important source of myofibroblasts that promote PF, while those in healthy subjects or under modified conditions may inhibit PF. Therefore, it is of great importance to determine the functional differences between BMSCs from normal physiological conditions and those from abnormal pathological conditions. The correct guidance and use of
BMSCs could improve health and promote their further use in clinical practices.

Research on BMSCs has a long history. It was expected that BMSCs would migrate to damaged organs and exert similar regenerative and differentiation effects as pluripotent stem cells to promote organ repair (Luo et al., 2019; Tang et al., 2020). However, the implantation rate of BMSCs has been shown to be extremely low in animal models, while their culture supernatant can also promote the repair of injured organs (Lai et al., 2010). Therefore, researchers have begun to focus on the paracrine mechanism of BMSCs. Recent studies have shown that cell-cell information exchange may occur through membrane EVs that are rich in bioactive factors, with exosomes, an EV subtype, have been thoroughly studied (Lai et al., 2010; Roy et al., 2018). It is currently believed that exosomes derived from BMSCs not only have a similar efficacy as BMSC transplantation but also reduce the risks associated with BMSC transplantation, such as immune rejection, teratogenic tumors, and pulmonary embolism (Lai et al., 2010; Mehryab et al., 2020; Peng et al., 2020; Qin et al., 2020). Thus, studies on the use of exosomes derived from BMSCs to repair the damaged organs are increasing.

Studies have reported that one week after BMSCs were used to treat myocardial ischemia and reperfusion injury, less than $1 \%$ of BMSCs survived and did not differentiate into cardiomyocytes, but they protected the heart through the paracrine mechanism mediated by exosomes (Lai et al., 2010). Exosomes derived from human umbilical cord MSCs can effectively alleviate liver fibrosis (Li et al., 2013). Exosomes show exciting therapeutic effects in lung injury and PF, showing similar or even better therapeutic potential as MSCs. BMSC conditioned medium can improve alveolar epithelial wound repair, inhibit alveolar epithelial mesenchymal transition, and significantly inhibit silicosis (Choi et al., 2014; Li et al., 2018b; Wang et al., 2018b). Mouse adipose-derived MSCs and their exosomes can improve PF and inflammation in advanced silicosis models, and high concentration of exosomes can achieve therapeutic effects that are comparable to MSCs (Bandeira et al., 2018). In a model of silicosis, BMSCs can migrate to damaged lung tissue, but cannot differentiate into alveolar epithelial cells. Instead, they promote the proliferation of type II alveolar epithelial cells, inhibit type II alveolar epithelial fibrosis, and inhibit collagen deposition through the paracrine pathway (Hostettler et al., 2017; Li et al., 2017; Wang et al., 2018b). Therefore, BMSCs have limited ability to self-differentiate; rather they primarily act through paracrine exosomes. However, the molecules and signaling pathways through which exosomes regulate silicosis remain unclear. 


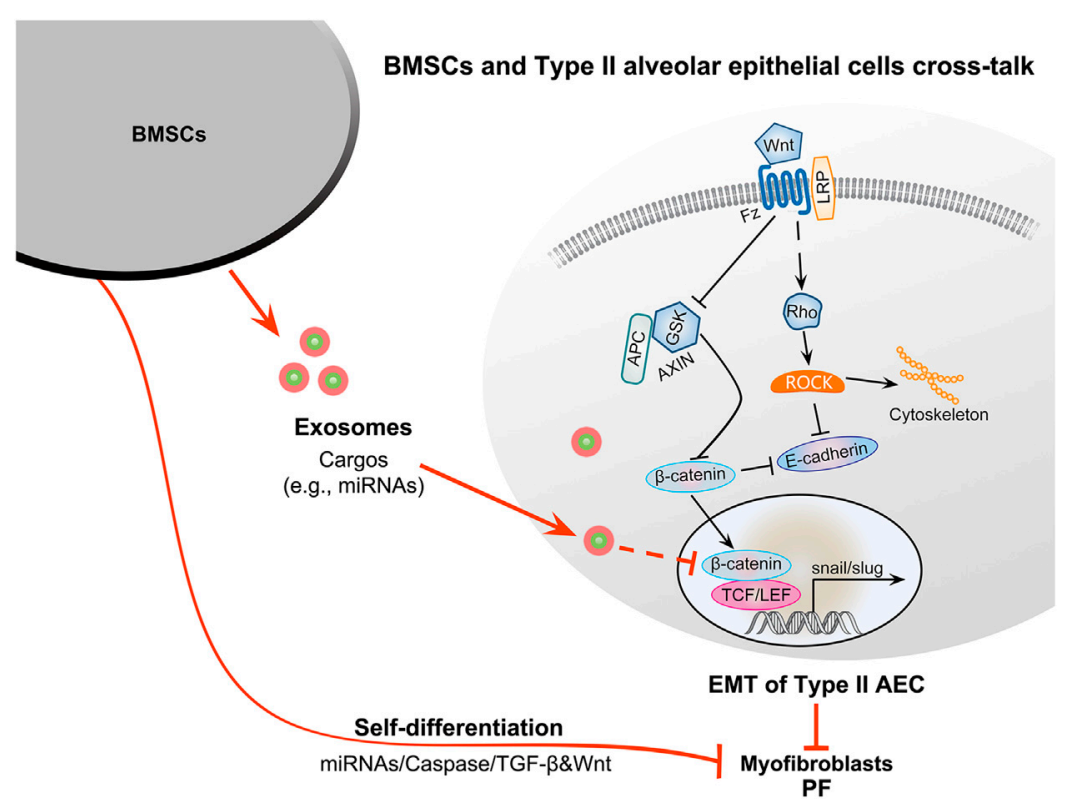

FIGURE 2| Therapeutic potential of BMSC-derived exosomes for PF and the potential molecular mechanisms. BMSCs in the PF microenvironment can contribute to myofibroblasts formation through self-differentiation and secrete exosomes to facilitate the EMT of type II alveolar epithelial cells to promote PF. In contrast, BMSCs from healthy individuals can migrate to damaged organs to promote their repair and secrete exosomes to inhibit the EMT of type II alveolar epithelial cells via the interplay between the Wnt and TGF- $\beta 1 /$ Smad signaling pathways. Exosomal cargos such as miRNAs can inhibit $\beta$-catenin and axin activity. Axin can negatively regulate the Wnt $\beta$-catenin signaling pathway, inhibiting the phosphorylation of GSK3 $\beta$ and leading to the suppression of $\beta$-catenin degradation. $\beta$-Catenin can promote the transformation of type II alveolar epithelial cells into myofibroblasts via EMT. Finally, the downregulation of axin can inhibit the EMT of type II alveolar epithelial cells to inhibit PF. The self-differentiation of BMSCs may be mediated by miRNAs, caspase, TGF- $\beta 1$, and Wnt signaling pathways.

Exosomes are the key factor regulating the differentiation of BMSCs into myofibroblasts. Exosomes from normal BMSCs may suppresses EMT by interacting with the $\mathrm{Wnt} / \beta$-catenin pathway in alveolar epithelial cells to inhibit PF (Figure 2).

\section{SUMMARY}

The occurrence of PF is closely associated with exosomes and the transition of epithelial cells into fibroblasts and mesenchymal cells (EMT), the transformation of fibroblasts, fibroblast proliferation, immunoregulatory effects, and mitochondrial damage. On the one hand, exosomes secreted by cells can affect the pathological processes of diseases by promoting the occurrence of EMT via their cargos, in which the microRNA contents are particularly relevant for the pathology of PF. For example, during the occurrence and development of $\mathrm{PF}$, exosomes can upregulate the EMT and enhance the differentiation of BMSCs into myofibroblasts. Therefore, a more in-depth study on the role of exosomes in fibroblast proliferation, fibroblast activation, and myofibroblast differentiation can provide new insights into PF diagnosis and treatment. On the other hand, future studies may identify a large number of exosomal molecules that can promote fibrosis inhibition, EMT inhibition, and even reverse the EMT process, which would have anti-fibrotic effects and alleviate the functional damage of fibrosis to human tissues and organs, since evidence supports their potential for altering gene programs and inducing the differentiation or dedifferentiation of target cells. Therefore, further research on the effects of exosomal cargos in the process of tissue fibrosis may provide a new method for the treatment of fibrosisrelated diseases. On the whole, with the continuous development of exosomal-related technologies, such as the isolation, identification, and modification of exosomes, exosomes may become a new direction in the research of drugs for the treatment of PF.

BMSCs have strong plasticity that acts as a double-edged sword. They can promote and antagonize fibrosis associated with the microenvironment changes. The differentiation and regulation of BMSCs is closed associated with exosomes. Exosomes with enriched specific cargos such as miR-214-3p may be used to treat silicosis by regulating the function of BMSCs and type II alveolar epithelial cells. Importantly, although BMSC-derived exosomes have a number of functions, such as in repairing tissue damage and suppressing inflammatory responses, the associated mechanisms are not fully understood, and the results remain controversial.

Most of the effects of exosomes observed in recent studies are from in vitro experiments, and only few cargos were identified, which should be investigated and validated in vivo in the future. Further in vivo and in vitro experimental studies to explore the molecular mechanism of BMSCs in the treatment of PF, to elucidate the therapeutic effects of BMSC-derived exosomes on the proliferation of type II alveolar epithelial cells and PF, and to reveal the regulatory mechanisms associated with $\mathrm{T} \beta \mathrm{R} 1$ signaling and the $\mathrm{Wnt} / \beta$-catenin pathway will provide a theoretical basis or new strategies for effectively terminating or reversing PF. 


\section{AUTHOR CONTRIBUTIONS}

YZ contributed to the conception and design of the work. All authors drafted the manuscript and approved the final manuscript.

\section{REFERENCES}

Ahuja, N., Ashok, C., Natua, S., Pant, D., Cherian, A., Pandkar, M. R., et al. (2020). Hypoxia-induced TGF- $\beta$-RBFOX2-ESRP1 axis regulates human MENA alternative splicing and promotes EMT in breast cancer. NAR Cancer 2 (3), zcaa021. doi:10.1093/narcan/zcaa021

Akbari, A., and Rezaie, J. (2020). Potential therapeutic application of mesenchymal stem cell-derived exosomes in SARS-CoV-2 pneumonia. Stem Cell Res. Ther. 11 (1), 356. doi:10.1186/s13287-020-01866-6

Bandeira, E., Oliveira, H., Silva, J. D., Menna-Barreto, R. F. S., Takyia, C. M., Suk, J. S., et al. (2018). Therapeutic effects of adipose-tissue-derived mesenchymal stromal cells and their extracellular vesicles in experimental silicosis. Respir. Res. 19 (1), 104. doi:10.1186/s12931-018-0802-3

Barnes, H., Goh, N. S. L., Leong, T. L., and Hoy, R. (2019). Silica-associated lung disease: an old-world exposure in modern industries. Respirology 24 (12), 1165-1175. doi:10.1111/resp.13695

Basile, D. P., and Mehrotra, P. (2017). Surprising enhancement of fibrosis by tubule-specific deletion of the TGF-beta receptor: a new twist on an old paradigm. J. Am. Soc. Nephrol. 28 (12), 3427-3429. doi:10.1681/asn. 2017080947

Bell, J. L., and Mazurek, J. M. (2020). Trends in pneumoconiosis deaths-United States, 1999-2018. MMWR Morb. Mortal. Wkly. Rep. 69 (23), 693-698. doi:10. 15585/mmwr.mm6923al

Budi, E. H., Duan, D., and Derynck, R. (2017). Transforming growth factor- $\beta$ receptors and Smads: regulatory complexity and functional versatility. Trends Cell Biol. 27 (9), 658-672. doi:10.1016/j.tcb.2017.04.005

Cai, X., Wang, L., Wang, X., and Hou, F. (2020). miR-124a enhances therapeutic effects of bone marrow stromal cells transplant on diabetic nephropathy-related epithelial-to-mesenchymal transition and fibrosis. J. Cell. Biochem. 121 (1), 299-312. doi:10.1002/jcb.29170

Cao, M., Seike, M., Soeno, C., Mizutani, H., Kitamura, K., Minegishi, Y., et al. (2012). MiR-23a regulates TGF- $\beta$-induced epithelial-mesenchymal transition by targeting E-cadherin in lung cancer cells. Int. J. Oncol. 41 (3), 869-875. doi:10.3892/ijo.2012.1535

Choi, M., Ban, T., and Rhim, T. (2014). Therapeutic use of stem cell transplantation for cell replacement or cytoprotective effect of microvesicle released from mesenchymal stem cell. Mol. Cells 37 (2), 133-139. doi:10.14348/molcells. 2014.2317

Chowdhury, R., Webber, J. P., Gurney, M., Mason, M. D., Tabi, Z., and Clayton, A. (2015). Cancer exosomes trigger mesenchymal stem cell differentiation into pro-angiogenic and pro-invasive myofibroblasts. Oncotarget 6 (2), 715-731. doi:10.18632/oncotarget.2711

Cocozza, F., Grisard, E., Martin-Jaular, L., Mathieu, M., and Théry, C. (2020). SnapShot: extracellular vesicles. Cell 182 (1), 262-262.e1. doi:10.1016/j.cell. 2020.04.054

Colombo, M., Raposo, G., and Théry, C. (2014). Biogenesis, secretion, and intercellular interactions of exosomes and other extracellular vesicles. Annu. Rev. Cell Dev. Biol. 30, 255-289. doi:10.1146/annurev-cellbio-101512-122326

Conigliaro, A., and Cicchini, C. (2018). Exosome-mediated signaling in epithelial to mesenchymal transition and tumor progression. J. Clin. Med. 8 (1), 26. doi: $10.3390 / \mathrm{jcm} 8010026$

Dai, W., Jin, X., Han, L., Huang, H., Ji, Z., Xu, X., et al. (2020). Exosomal lncRNA DOCK9-AS2 derived from cancer stem cell-like cells activated Wnt $/ \beta$-catenin pathway to aggravate stemness, proliferation, migration, and invasion in papillary thyroid carcinoma. Cell Death Dis. 11 (9), 743. doi:10.1038/ s41419-020-02827-w

DeMaio, L., Buckley, S. T., Krishnaveni, M. S., Flodby, P., Dubourd, M., Banfalvi, A., et al. (2012). Ligand-independent transforming growth factor- $\beta$ type I receptor signalling mediates type I collagen-induced epithelial-mesenchymal transition. J. Pathol. 226 (4), 633-644. doi:10.1002/path.3016

\section{FUNDING}

Funding was provided by the National Natural Science Foundation of China (Grant nos. 11932014 and 11402153).

Dinh, P. C., Paudel, D., Brochu, H., Popowski, K. D., Gracieux, M. C., Cores, J., et al (2020). Inhalation of lung spheroid cell secretome and exosomes promotes lung repair in pulmonary fibrosis. Nat. Commun. 11 (1), 1064. doi:10.1038/s41467020-14344-7

Donnarumma, E., Fiore, D., Nappa, M., Roscigno, G., Adamo, A., Iaboni, M., et al. (2017). Cancer-associated fibroblasts release exosomal microRNAs that dictate an aggressive phenotype in breast cancer. Oncotarget 8 (12), 19592-19608. doi:10.18632/oncotarget.14752

Du, Y., Xiao, H., Wan, J., Wang, X., Li, T., Zheng, S., et al. (2020). Atorvastatin attenuates TGF- $\beta 1$-induced fibrogenesis by inhibiting Smad3 and MAPK signaling in human ventricular fibroblasts. Int. J. Mol. Med. 46 (2), 633-640. doi:10.3892/ijmm.2020.4607

Edeling, M., Ragi, G., Huang, S., Pavenstadt, H., and Susztak, K. (2016). Developmental signalling pathways in renal fibrosis: the roles of Notch, Wnt and Hedgehog. Nat. Rev. Nephrol. 12 (7), 426-439. doi:10.1038/ nrneph.2016.54

Feng, Y. L., Chen, D. Q., Vaziri, N. D., Guo, Y., and Zhao, Y. Y. (2020). Small molecule inhibitors of epithelial-mesenchymal transition for the treatment of cancer and fibrosis. Med. Res. Rev. 40 (1), 54-78. doi:10. $1002 /$ med.21596

Fujita, Y., Araya, J., Ito, S., Kobayashi, K., Kosaka, N., Yoshioka, Y., et al. (2015) Suppression of autophagy by extracellular vesicles promotes myofibroblast differentiation in COPD pathogenesis. J. Extracell. Vesicles 4, 28388. doi:10. 3402/jev.v4.28388

Fukushima, K., Satoh, T., Sugihara, F., Sato, Y., Okamoto, T., Mitsui, Y., et al. (2020). Dysregulated expression of the nuclear exosome targeting complex component $\mathrm{Rbm} 7$ in nonhematopoietic cells licenses the development of fibrosis. Immunity 52 (3), 542-556. doi:10.1016/j.immuni.2020.02.007

Gross, T. J., and Hunninghake, G. W. (2001). Idiopathic pulmonary fibrosis. N. Engl. J. Med. 345 (7), 517-525. doi:10.1056/NEJMra003200

Guiot, J., Struman, I., Louis, E., Louis, R., Malaise, M., and Njock, M. S. (2019). Exosomal miRNAs in lung diseases: from biologic function to therapeutic targets. J. Clin. Med. 8 (9), 1345. doi:10.3390/jcm8091345

Guiot, J., Cambier, M., Boeckx, A., Henket, M., Nivelles, O., Gester, F., et al. (2020). Macrophage-derived exosomes attenuate fibrosis in airway epithelial cells through delivery of antifibrotic miR-142-3p. Thorax 75 (10), 870-881. doi:10.1136/thoraxjnl-2019-214077

Hayashi, H., and Sakai, T. (2012). Biological significance of local TGF- $\beta$ activation in liver diseases. Front. Physiol. 3, 12. doi:10.3389/fphys.2012.00012

He, S., Chen, D., Hu, M., Zhang, L., Liu, C., Traini, D., et al. (2019a). Bronchial epithelial cell extracellular vesicles ameliorate epithelial-mesenchymal transition in COPD pathogenesis by alleviating M2 macrophage polarization. Nanomedicine 18, 259-271. doi:10.1016/j.nano.2019.03.010

He, S., Li, Z., Yu, Y., Zeng, Q., Cheng, Y., Ji, W., et al. (2019b). Exosomal miR-499a$5 \mathrm{p}$ promotes cell proliferation, migration and EMT via mTOR signaling pathway in lung adenocarcinoma. Exp. Cell Res. 379 (2), 203-213. doi:10. 1016/j.yexcr.2019.03.035

Hirsova, P., Ibrahim, S. H., Krishnan, A., Verma, V. K., Bronk, S. F., Werneburg, N. W., et al. (2016). Lipid-induced signaling causes release of inflammatory extracellular vesicles from hepatocytes. Gastroenterology 150 (4), 956-967. doi:10.1053/j.gastro.2015.12.037

Hong, W., Xue, M., Jiang, J., Zhang, Y., and Gao, X. (2020). Circular RNA circCPA4/let-7 miRNA/PD-L1 axis regulates cell growth, stemness, drug resistance and immune evasion in non-small cell lung cancer (NSCLC). J. Exp. Clin. Cancer Res. 39 (1), 149. doi:10.1186/s13046-020-01648-1

Hopkins, R. B., Burke, N., Fell, C., Dion, G., and Kolb, M. (2016). Epidemiology and survival of idiopathic pulmonary fibrosis from national data in Canada. Eur. Respir. J. 48 (1), 187-195. doi:10.1183/13993003.01504-2015

Hostettler, K. E., Gazdhar, A., Khan, P., Savic, S., Tamo, L., Lardinois, D., et al. (2017). Multipotent mesenchymal stem cells in lung fibrosis. PLoS One 12 (8), e0181946. doi:10.1371/journal.pone.0181946 
Hoy, R. F., and Chambers, D. C. (2020). Silica-related diseases in the modern world. Allergy 75 (11), 3011. doi:10.1111/all.14202

Hu, Y. B., Li, X., Liang, G. N., Deng, Z. H., Jiang, H. Y., and Zhou, J. H. (2013). Roles of Rho/Rock signaling pathway in silica-induced epithelial-mesenchymal transition in human bronchial epithelial cells. Biomed. Environ. Sci. 26 (7), 571-576. doi:10.3967/0895-3988.2013.07.008

Hu, J., Chen, Y., Huang, Y., and Su, Y. (2020). Human umbilical cord mesenchymal stem cell-derived exosomes suppress dermal fibroblasts-myofibroblats transition via inhibiting the TGF- $\beta 1 /$ Smad 2/3 signaling pathway. Exp. Mol. Pathol. 115, 104468. doi:10.1016/j.yexmp.2020.104468

Ijaz, T., Pazdrak, K., Kalita, M., Konig, R., Choudhary, S., Tian, B., et al. (2014). Systems biology approaches to understanding Epithelial Mesenchymal Transition (EMT) in mucosal remodeling and signaling in asthma. World Allergy Organ J. 7 (1), 13. doi:10.1186/1939-4551-7-13

Jia, Z., Johnson, A. C., Wang, X., Guo, Z., Dreisbach, A. W., Lewin, J. R., et al. (2015). Allelic variants in Arhgef11 via the Rho-ROCK pathway are linked to epithelial-mesenchymal transition and contributes to kidney injury in the dahl salt-sensitive rat. PLoS One 10 (7), e0132553. doi:10.1371/journal.pone.0132553

Jin, J., Wang, Y., Zhao, L., Zou, W., Tan, M., and He, Q. (2020). Exosomal miRNA215-5p derived from adipose-derived stem cells attenuates epithelialmesenchymal transition of podocytes by inhibiting ZEB2. BioMed Res. Int. 2020, 2685305. doi:10.1155/2020/2685305

Johan, M. Z., and Samuel, M. S. (2019). Rho-ROCK signaling regulates tumormicroenvironment interactions. Biochem. Soc. Trans. 47 (1), 101-108. doi:10. 1042/bst20180334

Kadota, T., Yoshioka, Y., Fujita, Y., Araya, J., Minagawa, S., Hara, H., et al. (2020). Extracellular vesicles from fibroblasts induce epithelial cell senescence in pulmonary fibrosis. Am. J. Respir. Cell Mol. Biol. 63 (5), 623-636. doi:10. 1165/rcmb.2020-0002OC

Kang, J. S., Liu, C., and Derynck, R. (2009). New regulatory mechanisms of TGFbeta receptor function. Trends Cell Biol. 19 (8), 385-394. doi:10.1016/j.tcb.2009. 05.008

Keller, S., Ridinger, J., Rupp, A. K., Janssen, J. W., and Altevogt, P. (2011). Body fluid derived exosomes as a novel template for clinical diagnostics. J. Transl. Med. 9, 86. doi:10.1186/1479-5876-9-86

Khalaj, K., Figueira, R. L., Antounians, L., Lauriti, G., and Zani, A. (2020). Systematic review of extracellular vesicle-based treatments for lung injury: are EVs a potential therapy for COVID-19?. J. Extracell. Vesicles 9 (1), 1795365. doi:10.1080/20013078.2020.1795365

Kim, J., Kim, T. Y., Lee, M. S., Mun, J. Y., Ihm, C., and Kim, S. A. (2016). Exosome cargo reflects TGF- $\beta 1$-mediated epithelial-to-mesenchymal transition (EMT) status in A549 human lung adenocarcinoma cells. Biochem. Biophys. Res. Commun. 478 (2), 643-648. doi:10.1016/j.bbrc.2016.07.124

Kim, Y. B., Ahn, Y. H., Jung, J. H., Lee, Y. J., Lee, J. H., and Kang, J. L. (2019). Programming of macrophages by UV-irradiated apoptotic cancer cells inhibits cancer progression and lung metastasis. Cell. Mol. Immunol. 16 (11), 851-867. doi:10.1038/s41423-019-0209-1

King, T. E., Jr., Pardo, A., and Selman, M. (2011). Idiopathic pulmonary fibrosis. Lancet 378 (9807), 1949-1961. doi:10.1016/s0140-6736(11)60052-4

Kinoshita, T., and Goto, T. (2019). Molecular mechanisms of pulmonary fibrogenesis and its progression to lung cancer: a review. Int. J. Mol. Sci. 20 (6), 1461. doi:10.3390/ijms20061461

Kiszalkiewicz, J., Piotrowski, W., and Brzezianska-Lasota, E. (2017). Signaling pathways and their miRNA regulators involved in the etiopathology of idiopathic pulmonary fibrosis (IPF) and hypersensitivity pneumonitis (HP). Adv. Respir. Med. 85 (3), 169-178. doi:10.5603/ARM.2017.0029

Kohn, A. D., and Moon, R. T. (2005). Wnt and calcium signaling: beta-cateninindependent pathways. Cell Calcium 38 (3-4), 439-446. doi:10.1016/j.ceca.2005. 06.022

Kropski, J. A., and Blackwell, T. S. (2019). Progress in understanding and treating idiopathic pulmonary fibrosis. Annu. Rev. Med. 70, 211-224. doi:10.1146/ annurev-med-041317-102715

Kumari, A., Dash, D., and Singh, R. (2017). Curcumin inhibits lipopolysaccharide (LPS)-induced endotoxemia and airway inflammation through modulation of sequential release of inflammatory mediators (TNF- $\alpha$ and TGF- $\beta 1$ ) in murine model. Inflammopharmacology 25 (3), 329-341. doi:10.1007/s10787-017-0334-3

Kuse, N., Kamio, K., Azuma, A., Matsuda, K., Inomata, M., Usuki, J., et al. (2020). Exosome-derived microRNA-22 ameliorates pulmonary fibrosis by regulating fibroblast-to-myofibroblast differentiation in vitro and in vivo. J. Nippon Med. Sch. 87 (3), 118-128. doi:10.1272/jnms.JNMS.2020_87-302

Lacy, S. H., Woeller, C. F., Thatcher, T. H., Pollock, S. J., Small, E. M., Sime, P. J., et al. (2019). Activated human lung fibroblasts produce extracellular vesicles with antifibrotic prostaglandins. Am. J. Respir. Cell Mol. Biol. 60 (3), 269-278. doi:10.1165/rcmb.2017-0248OC

Lai, R. C., Arslan, F., Lee, M. M., Sze, N. S., Choo, A., Chen, T. S., et al. (2010). Exosome secreted by MSC reduces myocardial ischemia/reperfusion injury. Stem Cell Res. 4 (3), 214-222. doi:10.1016/j.scr.2009.12.003

LeBleu, V. S., Taduri, G., O’Connell, J., Teng, Y., Cooke, V. G., Woda, C., et al. (2013). Origin and function of myofibroblasts in kidney fibrosis. Nat. Med. 19 (8), 1047-1053. doi:10.1038/nm.3218

Li, T., Yan, Y., Wang, B., Qian, H., Zhang, X., Shen, L., et al. (2013). Exosomes derived from human umbilical cord mesenchymal stem cells alleviate liver fibrosis. Stem Cells Dev. 22 (6), 845-854. doi:10.1089/scd.2012.0395

Li, X., Wang, Y., An, G., Liang, D., Zhu, Z., Lian, X., et al. (2017). Bone marrow mesenchymal stem cells attenuate silica-induced pulmonary fibrosis via paracrine mechanisms. Toxicol. Lett. 270, 96-107. doi:10.1016/j.toxlet.2017. 02.016

Li, M., Qiu, L., Hu, W., Deng, X., Xu, H., Cao, Y., et al. (2018a). Geneticallymodified bone mesenchymal stem cells with TGF- $\beta(3)$ improve wound healing and reduce scar tissue formation in a rabbit model. Exp. Cell Res. 367 (1), 24-29. doi:10.1016/j.yexcr.2018.02.006

Li, X., An, G., Wang, Y., Liang, D., Zhu, Z., and Tian, L. (2018b). Targeted migration of bone marrow mesenchymal stem cells inhibits silica-induced pulmonary fibrosis in rats. Stem Cell Res. Ther. 9 (1), 335. doi:10.1186/s13287018-1083-y

Li, J., Liu, J., Yue, W., Xu, K., Cai, W., Cui, F., et al. (2020a). Andrographolide attenuates epithelial-mesenchymal transition induced by TGF- $\beta 1$ in alveolar epithelial cells. J. Cell Mol. Med. 24 (18), 10501-10511. doi:10.1111/jcmm.15665

Li, K., Liu, T., Chen, J., Ni, H., and Li, W. (2020b). Survivin in breast cancer-derived exosomes activates fibroblasts by upregulating SOD1, whose feedback promotes cancer proliferation and metastasis. J. Biol. Chem. 295 (40), 13737-13752. doi:10.1074/jbc.RA120.013805

Li, N., Li, J., Mi, Q., Xie, Y., Li, P., Wang, L., et al. (2020c). Long non-coding RNA ADAMTS9-AS1 suppresses colorectal cancer by inhibiting the $\mathrm{Wnt} / \beta$-catenin signalling pathway and is a potential diagnostic biomarker. J. Cell Mol. Med. 24 (19), 11318-11329. doi:10.1111/jcmm.15713

Li, T., Wan, Y., Su, Z., Li, J., Han, M., and Zhou, C. (2020d). Mesenchymal stem cell-derived exosomal microRNA-3940-5p inhibits colorectal cancer metastasis by targeting integrin a6. Dig. Dis. Sci. doi:10.1007/s10620-020-06458-1

Liang, H., Gu, Y., Li, T., Zhang, Y., Huangfu, L., Hu, M., et al. (2014). Integrated analyses identify the involvement of microRNA-26a in epithelial-mesenchymal transition during idiopathic pulmonary fibrosis. Cell Death Dis. 5 (5), e1238. doi:10.1038/cddis.2014.207

Lichtman, M. K., Otero-Vinas, M., and Falanga, V. (2016). Transforming growth factor beta (TGF- $\beta$ ) isoforms in wound healing and fibrosis. Wound Repair Regen. 24 (2), 215-222. doi:10.1111/wrr.12398

Liu, Y., Steenland, K., Rong, Y., Hnizdo, E., Huang, X., Zhang, H., et al. (2013). Exposure-response analysis and risk assessment for lung cancer in relationship to silica exposure: a 44 -year cohort study of 34,018 workers. Am. J. Epidemiol. 178 (9), 1424-1433. doi:10.1093/aje/kwt139

Liu, W. W., Wang, H. X., Yu, W., Bi, X. Y., Chen, J. Y., Chen, L. Z., et al. (2015). Treatment of silicosis with hepatocyte growth factor-modified autologous bone marrow stromal cells: a non-randomized study with follow-up. Genet. Mol. Res. 14 (3), 10672-10681. doi:10.4238/2015.September.9.7

Liu, S., Rong, G., Li, X., Geng, L., Zeng, Z., Jiang, D., et al. (2020). Diosgenin and GSK126 produce synergistic effects on epithelial-mesenchymal transition in gastric cancer cells by mediating EZH2 via the Rho/ROCK signaling pathway. OncoTargets Ther. 13, 5057-5067. doi:10.2147/OTT.S237474

Lobb, R. J., van Amerongen, R., Wiegmans, A., Ham, S., Larsen, J. E., and Möller, A. (2017). Exosomes derived from mesenchymal non-small cell lung cancer cells promote chemoresistance. Int. J. Cancer 141 (3), 614-620. doi:10.1002/ijc. 30752

Luo, Z. W., Li, F. X., Liu, Y. W., Rao, S. S., Yin, H., Huang, J., et al. (2019). Aptamerfunctionalized exosomes from bone marrow stromal cells target bone to promote bone regeneration. Nanoscale 11 (43), 20884-20892. doi:10.1039/ c9nr02791b 
Luo, F., Xu, R., Song, G., Xue, D., He, X., and Xia, Y. (2020). Alleviation of TGF- $\beta 1$ induced tubular epithelial-mesenchymal transition via the $\delta$-opioid receptor. FEBS J. doi:10.1111/febs.15459

Macias, M. J., Martin-Malpartida, P., and Massagué, J. (2015). Structural determinants of Smad function in TGF- $\beta$ signaling. Trends Biochem. Sci. 40 (6), 296-308. doi:10.1016/j.tibs.2015.03.012

Mansouri, N., Willis, G. R., Fernandez-Gonzalez, A., Reis, M., Nassiri, S., Mitsialis, S. A., et al. (2019). Mesenchymal stromal cell exosomes prevent and revert experimental pulmonary fibrosis through modulation of monocyte phenotypes. JCI Insight 4 (21), e128060. doi:10.1172/jci.insight.128060

Martin-Medina, A., Lehmann, M., Burgy, O., Hermann, S., Baarsma, H. A., Wagner, D. E., et al. (2018). Increased extracellular vesicles mediate WNT5A signaling in idiopathic pulmonary fibrosis. Am. J. Respir. Crit. Care Med. 198 (12), 1527-1538. doi:10.1164/rccm.201708-1580OC

Mathieu, M., Martin-Jaular, L., Lavieu, G., and Théry, C. (2019). Specificities of secretion and uptake of exosomes and other extracellular vesicles for cell-to-cell communication. Nat. Cell Biol. 21 (1), 9-17. doi:10.1038/s41556-018-0250-9

Mehryab, F., Rabbani, S., Shahhosseini, S., Shekari, F., Fatahi, Y., Baharvand, H., et al. (2020). Exosomes as a next-generation drug delivery system: an update on drug loading approaches, characterization, and clinical application challenges. Acta Biomater. 113, 42-62. doi:10.1016/j.actbio.2020.06.036

Meyer, K. C. (2017). Pulmonary fibrosis, part I: epidemiology, pathogenesis, and diagnosis. Expert Rev Respir Med 11 (5), 343-359. doi:10.1080/17476348.2017. 1312346

Moghiman, T., Barghchi, B., Esmaeili, S. A., Shabestari, M. M., Tabaee, S. S., and Momtazi-Borojeni, A. A. (2020). Therapeutic angiogenesis with exosomal microRNAs: an effectual approach for the treatment of myocardial ischemia. Heart Fail. Rev. doi:10.1007/s10741-020-10001-9

Ng-Blichfeldt, J. P., de Jong, T., Kortekaas, R. K., Wu, X., Lindner, M., Guryev, V., et al. (2019). TGF- $\beta$ activation impairs fibroblast ability to support adult lung epithelial progenitor cell organoid formation. Am. J. Physiol. Lung Cell Mol. Physiol. 317 (1), L14-128. doi:10.1152/ajplung.00400.2018

Nieto, M. A., Huang, R. Y., Jackson, R. A., and Thiery, J. P. (2016). EMT: 2016. Cell 166 (1), 21-45. doi:10.1016/j.cell.2016.06.028

Nlandu-Khodo, S., Neelisetty, S., Phillips, M., Manolopoulou, M., Bhave, G., May, L., et al. (2017). Blocking TGF- $\beta$ and $\beta$-catenin epithelial crosstalk exacerbates CKD. J. Am. Soc. Nephrol. 28 (12), 3490-3503. doi:10.1681/asn.2016121351

Pan, R., Zhang, Y., Zheng, M., Zang, B., and Jin, M. (2017). Hydroxysafflor yellow A suppresses MRC- 5 cell activation induced by TGF- $\beta 1$ by blocking TGF- $\beta 1$ binding to T $\beta$ RII. Front. Pharmacol. 8, 264. doi:10.3389/fphar.2017.00264

Peng, H., Ji, W., Zhao, R., Yang, J., Lu, Z., Li, Y., et al. (2020). Exosome: a significant nano-scale drug delivery carrier. J. Mater. Chem. B. 8 (34), 7591-7608. doi:10. 1039/d0tb01499k

Porazinski, S., Parkin, A., and Pajic, M. (2020). Rho-ROCK signaling in normal physiology and as a key player in shaping the tumor microenvironment. $A d v$. Exp. Med. Biol. 1223, 99-127. doi:10.1007/978-3-030-35582-1_6

Qian, W., Cai, X., Qian, Q., Zhang, W., and Tian, L. (2020). Metastasis-associated protein 1 promotes epithelial-mesenchymal transition in idiopathic pulmonary fibrosis by up-regulating Snail expression. J. Cell Mol. Med. 24 (11), 5998-6007. doi:10.1111/jcmm.15062

Qin, X. J., Zhang, J. X., and Wang, R. L. (2020). Exosomes as mediators and biomarkers in fibrosis. Biomark. Med. 14 (8), 697-712. doi:10.2217/bmm-2019-0368

Rout-Pitt, N., Farrow, N., Parsons, D., and Donnelley, M. (2018). Epithelial mesenchymal transition (EMT): a universal process in lung diseases with implications for cystic fibrosis pathophysiology. Respir. Res. 19 (1), 136. doi:10.1186/s12931-018-0834-8

Roy, S., Hochberg, F. H., and Jones, P. S. (2018). Extracellular vesicles: the growth as diagnostics and therapeutics; a survey. J. Extracell. Vesicles 7 (1), 1438720. doi:10.1080/20013078.2018.1438720

Sakurai, R., Liu, J., Wang, Y., Torday, J. S., and Rehan, V. K. (2018). Prevention of perinatal nicotine-induced bone marrow mesenchymal stem cell myofibroblast differentiation by augmenting the lipofibroblast phenotype. Clin. Sci. (Lond.) 132 (21), 2357-2368. doi:10.1042/cs20180749

Scavo, M. P., Rizzi, F., Depalo, N., Fanizza, E., Ingrosso, C., Curri, M. L., et al. (2020). A possible role of FZD10 delivering exosomes derived from colon cancers cell lines in inducing activation of epithelial-mesenchymal transition in normal colon epithelial cell line. Int. J. Mol. Sci. 21 (18), 6705. doi:10.3390/ ijms 21186705
Shi, Y., and Massagué, J. (2003). Mechanisms of TGF-beta signaling from cell membrane to the nucleus. Cell 113 (6), 685-700. doi:10.1016/s0092-8674(03) 00432-x

Singh, M., Yelle, N., Venugopal, C., and Singh, S. K. (2018). EMT: mechanisms and therapeutic implications. Pharmacol. Ther. 182, 80-94. doi:10.1016/j. pharmthera.2017.08.009

Stahl, P. D., and Raposo, G. (2019). Extracellular vesicles: exosomes and microvesicles, integrators of homeostasis. Physiology 34 (3), 169-177. doi:10. 1152/physiol.00045.2018

Sun, Z., Wang, C., Shi, C., Sun, F., Xu, X., Qian, W., et al. (2014). Activated Wnt signaling induces myofibroblast differentiation of mesenchymal stem cells, contributing to pulmonary fibrosis. Int. J. Mol. Med. 33 (5), 1097-1109. doi:10. 3892/ijmm.2014.1672

Sun, L., Zhu, M., Feng, W., Lin, Y., Yin, J., Jin, J., et al. (2019). Exosomal miRNA let7 from menstrual blood-derived endometrial stem cells alleviates pulmonary fibrosis through regulating mitochondrial DNA damage. Oxid Med Cell Longev. 2019, 4506303, doi:10.1155/2019/4506303

Suryadevara, V., Ramchandran, R., Kamp, D. W., and Natarajan, V. (2020). Lipid mediators regulate pulmonary fibrosis: potential mechanisms and signaling pathways. Int. J. Mol. Sci. 21 (12), 4257. doi:10.3390/ijms21124257

Syn, N., Wang, L., Sethi, G., Thiery, J. P., and Goh, B. C. (2016). Exosome-mediated metastasis: from epithelial-mesenchymal transition to escape from immunosurveillance. Trends Pharmacol. Sci. 37 (7), 606-617. doi:10.1016/j. tips.2016.04.006

Tang, Y. T., Huang, Y. Y., Li, J. H., Qin, S. H., Xu, Y., An, T. X., et al. (2018). Alterations in exosomal miRNA profile upon epithelial-mesenchymal transition in human lung cancer cell lines. BMC Genom. 19 (1), 802. doi:10. 1186/s12864-018-5143-6

Tang, Y., Chen, C., Liu, F., Xie, S., Qu, J., Li, M., et al. (2020). Structure and ingredient-based biomimetic scaffolds combining with autologous bone marrow-derived mesenchymal stem cell sheets for bone-tendon healing. Biomaterials 241, 119837. doi:10.1016/j.biomaterials.2020.119837

Teeuwssen, M., and Fodde, R. (2019). Wnt signaling in ovarian cancer stemness, EMT, and therapy resistance. J. Clin. Med. 8 (10). doi:10.3390/jcm8101658

Tellez, C. S., Juri, D. E., Do, K., Bernauer, A. M., Thomas, C. L., Damiani, L. A., et al. (2011). EMT and stem cell-like properties associated with miR-205 and miR200 epigenetic silencing are early manifestations during carcinogen-induced transformation of human lung epithelial cells. Cancer Res 71 (8), 3087-3097. doi:10.1158/0008-5472.Can-10-3035

Thannickal, V. J., Toews, G. B., White, E. S., Lynch, J. P., 3rd, and Martinez, F. J. (2004). Mechanisms of pulmonary fibrosis. Annu. Rev. Med. 55, 395-417. doi:10.1146/annurev.med.55.091902.103810

van Niel, G., D'Angelo, G., and Raposo, G. (2018). Shedding light on the cell biology of extracellular vesicles. Nat. Rev. Mol. Cell Biol. 19 (4), 213-228. doi:10. 1038/nrm.2017.125

Wan, F. Z., Chen, K. H., Sun, Y. C., Chen, X. C., Liang, R. B., Chen, L., et al. (2020). Exosomes overexpressing miR-34c inhibit malignant behavior and reverse the radioresistance of nasopharyngeal carcinoma. J. Transl. Med. 18 (1), 12. doi:10. 1186/s12967-019-02203-z

Wang, L., Yang, L., Tian, L., Mai, P., Jia, S., Yang, L., et al. (2017). Cannabinoid receptor 1 mediates homing of bone marrow-derived mesenchymal stem cells triggered by chronic liver injury. J. Cell. Physiol. 232 (1), 110-121. doi:10.1002/ jcp. 25395

Wang, J. N., Li, L., Li, L. Y., Yan, Q., Li, J., and Xu, T. (2018a). Emerging role and therapeutic implication of Wnt signaling pathways in liver fibrosis. Gene 674, 57-69. doi:10.1016/j.gene.2018.06.053

Wang, X., Gao, J. L., Zhao, M. M., Zhu, H. X., Tian, Y. X., Li, R., et al. (2018b). Therapeutic effects of conditioned medium from bone marrow-derived mesenchymal stem cells on epithelial-mesenchymal transition in A549 cells. Int. J. Mol. Med. 41 (2), 659-668. doi:10.3892/ijmm.2017.3284

Wang, D., Hao, C., Zhang, L., Zhang, J., Liu, S., Li, Y., et al. (2020). Exosomal miR$125 \mathrm{a}-5 \mathrm{p}$ derived from silica-exposed macrophages induces fibroblast transdifferentiation. Ecotoxicol. Environ. Saf. 192, 110253. doi:10.1016/j. ecoenv.2020.110253

Wen, C., Seeger, R. C., Fabbri, M., Wang, L., Wayne, A. S., and Jong, A. Y. (2017). Biological roles and potential applications of immune cell-derived extracellular vesicles. J. Extracell. Vesicles 6 (1), 1400370. doi:10.1080/20013078.2017. 1400370 
Willis, G. R., Fernandez-Gonzalez, A., Anastas, J., Vitali, S. H., Liu, X., Ericsson, M., et al. (2018). Mesenchymal stromal cell exosomes ameliorate experimental bronchopulmonary dysplasia and restore lung function through macrophage immunomodulation. Am. J. Respir. Crit. Care Med. 197 (1), 104-116. doi:10. 1164/rccm.201705-0925OC

Wodarz, A., and Nusse, R. (1998). Mechanisms of Wnt signaling in development. Annu. Rev. Cell Dev. Biol. 14, 59-88. doi:10.1146/annurev.cellbio.14.1.59

Worthington, E. N., and Hagood, J. S. (2020). Therapeutic use of extracellular vesicles for acute and chronic lung disease. Int. J. Mol. Sci. 21 (7). doi:10.3390/ ijms 21072318

Wynn, T. A., (2011). Integrating mechanisms of pulmonary fibrosis. J. Exp. Med. 208 (7), 1339-1350. doi:10.1084/jem.20110551

Xiao, D., Barry, S., Kmetz, D., Egger, M., Pan, J., Rai, S. N., et al. (2016). Melanoma cell-derived exosomes promote epithelial-mesenchymal transition in primary melanocytes through paracrine/autocrine signaling in the tumor microenvironment. Cancer Lett. 376 (2), 318-327. doi:10.1016/j.canlet.2016. 03.050

Xu, Z., Ding, W., and Deng, X. (2019). PM(2.5), fine particulate matter: a novel player in the epithelial-mesenchymal transition?. Front. Physiol. 10, 1404. doi:10.3389/fphys.2019.01404

Yan, Z., Liu, J., Xie, L., Liu, X., and Zeng, Y. (2016). Role of heparan sulfate in mediating CXCL8-induced endothelial cell migration. PeerJ 4, e1669. doi:10. 7717/peerj.1669

Yanagihara, T., Chong, S. G., Vierhout, M., Hirota, J. A., Ask, K., and Kolb, M. (2020). Current models of pulmonary fibrosis for future drug discovery efforts. Expert Opin. Drug Discov. 15 (8), 931-941. doi:10.1080/17460441.2020.1755252

Yang, L., Chang, N., Liu, X., Han, Z., Zhu, T., Li, C., et al. (2012). Bone marrowderived mesenchymal stem cells differentiate to hepatic myofibroblasts by transforming growth factor- $\beta 1$ via sphingosine kinase/sphingosine 1phosphate (S1P)/S1P receptor axis. Am. J. Pathol. 181 (1), 85-97. doi:10. 1016/j.ajpath.2012.03.014

Yang, Y., Hong, Y., Cho, E., Kim, G. B., and Kim, I. S. (2018). Extracellular vesicles as a platform for membrane-associated therapeutic protein delivery. J. Extracell. Vesicles 7 (1), 1440131. doi:10.1080/20013078.2018.1440131

Yang, F., Yan, Y., Yang, Y., Hong, X., Wang, M., Yang, Z., et al. (2020). MiR-210 in exosomes derived from CAFs promotes non-small cell lung cancer migration and invasion through PTEN/PI3K/AKT pathway. Cell. Signal. 73, 109675. doi:10.1016/j.cellsig.2020.109675

Yao, M. Y., Zhang, W. H., Ma, W. T., Liu, Q. H., Xing, L. H., and Zhao, G. F. (2019a). microRNA-328 in exosomes derived from M2 macrophages exerts a promotive effect on the progression of pulmonary fibrosis via FAM13A in a rat model. Exp. Mol. Med. 51 (6), 1-16. doi:10.1038/s12276-019-0255-x

Yao, Y., Chen, R., Wang, G., Zhang, Y., and Liu, F. (2019b). Exosomes derived from mesenchymal stem cells reverse EMT via TGF- $\beta 1 /$ Smad pathway and promote repair of damaged endometrium. Stem Cell Res. Ther. 10 (1), 225. doi:10.1186/ s13287-019-1332-8

Yao, X., Xie, L., and Zeng, Y. (2020). MiR-9 promotes angiogenesis via targeting on sphingosine-1-phosphate receptor 1. Front Cell Dev. Biol. 8, 755. doi:10.3389/ fcell.2020.00755

Ye, C., Li, H., Bao, M., Zhuo, R., Jiang, G., and Wang, W. (2020). Alveolar macrophage-derived exosomes modulate severity and outcome of acute lung injury. Aging 12 (7), 6120-6128. doi:10.18632/aging.103010
Yeh, Y., Guo, Q., Connelly, Z., Cheng, S., Yang, S., Prieto-Dominguez, N., et al (2019). Wnt/beta-catenin signaling and prostate cancer therapy resistance. $A d v$. Exp. Med. Biol. 1210, 351-378. doi:10.1007/978-3-030-32656-2_16

Yoon, Y. S., Lee, Y. J., Choi, Y. H., Park, Y. M., and Kang, J. L. (2016). Macrophages programmed by apoptotic cells inhibit epithelial-mesenchymal transition in lung alveolar epithelial cells via PGE2, PGD2, and HGF. Sci. Rep. 6, 20992. doi:10.1038/srep20992

You, J., Li, M., Cao, L. M., Gu, Q. H., Deng, P. B., Tan, Y., et al. (2019). Snail1dependent cancer-associated fibroblasts induce epithelial-mesenchymal transition in lung cancer cells via exosomes. QJM 112 (8), 581-590. doi:10. 1093/qjmed/hcz093

Zeng, Y., Yao, X., Liu, X., He, X., Li, L., Liu, X., et al. (2019). Anti-angiogenesis triggers exosomes release from endothelial cells to promote tumor vasculogenesis. J. Extracell. Vesicles 8 (1), 1629865. doi:10.1080/20013078. 2019.1629865

Zhang, J., Tian, X. J., and Xing, J. (2016). Signal transduction pathways of EMT induced by TGF-beta, SHH, and WNT and their crosstalks. J. Clin. Med. 5 (4), 41. doi: $10.3390 / \mathrm{jcm} 5040041$

Zhang, X., Sai, B., Wang, F., Wang, L., Wang, Y., Zheng, L., et al. (2019). Hypoxic BMSC-derived exosomal miRNAs promote metastasis of lung cancer cells via STAT3-induced EMT. Mol. Cancer 18 (1), 40. doi:10.1186/s12943-019-0959-5

Zhang, X., Bai, J., Hang, Y., Long, L., Zheng, Z., Wang, Q., et al. (2020). Exosomal miR-1255b-5p targets human telomerase reverse transcriptase in colorectal cancer cells to suppress epithelial-to-mesenchymal transition. Mol Oncol. 14 (10), 2589-2608. doi:10.1002/1878-0261.12765

Zhao, J., Shi, W., Wang, Y. L., Chen, H., Bringas, P., Jr., Datto, M. B., et al. (2002). Smad3 deficiency attenuates bleomycin-induced pulmonary fibrosis in mice. Am. J. Physiol. Lung Cell Mol. Physiol. 282 (3), L585-L593. doi:10.1152/ajplung. 00151.2001

Zhao, X., Wu, X., Qian, M., Song, Y., Wu, D., and Zhang, W. (2018). Knockdown of TGF- $\beta 1$ expression in human umbilical cord mesenchymal stem cells reverts their exosome-mediated EMT promoting effect on lung cancer cells. Cancer Lett. 428, 34-44. doi:10.1016/j.canlet.2018.04.026

Zhao, F. Y., Cheng, T. Y., Yang, L., Huang, Y. H., Li, C., Han, J. Z., et al. (2020). G-CSF inhibits pulmonary fibrosis by promoting BMSC homing to the lungs via SDF-1/CXCR4 chemotaxis. Sci. Rep. 10 (1), 10515. doi:10.1038/s41598-02065580-2

Zuo, L., Xie, Y., Tang, J., Xin, S., Liu, L., Zhang, S., et al. (2019). Targeting exosomal EBV-LMP1 transfer and miR-203 expression via the NF- $\kappa B$ pathway: the therapeutic role of aspirin in NPC. Mol. Ther. Nucl. Acids 17, 175-184. doi:10.1016/j.omtn.2019.05.023

Conflict of Interest: The authors declare that the research was conducted in the absence of any commercial or financial relationships that could be construed as a potential conflict of interest.

Copyright () 2020 Xie and Zeng. This is an open-access article distributed under the terms of the Creative Commons Attribution License (CC BY). The use, distribution or reproduction in other forums is permitted, provided the original author(s) and the copyright owner(s) are credited and that the original publication in this journal is cited, in accordance with accepted academic practice. No use, distribution or reproduction is permitted which does not comply with these terms. 